Revista Brasileira de Cartografia

ISSN 1808-0936 | https://doi.org/10.14393/revbrascartogr

Sociedade Brasileira de Cartografia, Geodésia, Fotogrametria e Sensoriamento Remoto

\title{
Melhoria no limiar de detecção de perda de ciclos para o método das TD no posicionamento relativo
}

\author{
Improvement of cycles slip threshold for the TD method in the relative positioning
}

Caio Henrique Chrisóstomo Mendonça ${ }^{1}$, Daniele Barrocá Marra Alves ${ }^{2}$ e Crislaine Menezes da Silva ${ }^{3}$

1 Universidade Estadual Paulista (FCT/Unesp), Programa de Pós-graduação em Ciências Cartográficas, Presidente Prudente, São

Paulo. caiomendonca94@gmail.com.

ORCID: https://orcid.org/0000-0001-7984-5759

2 Universidade Estadual Paulista (FCT/Unesp), Departamento de Cartografia, Presidente Prudente, São Paulo.

daniele.barroca@unesp.br.

ORCID: https://orcid.org/0000-0002-9033-8499

3 Universidade Estadual Paulista (FCT/Unesp), Programa de Pós-graduação em Ciências Cartográficas, Presidente Prudente, São

Paulo. smenezescrislaine@gmail.com.

ORCID: https://orcid.org/0000-0003-2023-4237

Resumo: A observável fase da onda portadora, juntamente com a pseudodistância, são empregadas em posicionamento de alta acurácia no posicionamento por sistemas globais de navegação por satélite. Mas, a observável fase pode sofrer perda de ciclos durante o rastreamento do sinal. A perda de ciclos é uma descontinuidade do número inteiro de ciclos na fase da onda portadora, causada por uma perda temporária de rastreamento do sinal pelo receptor. Assim, as perdas de ciclos devem ser detectadas e corrigidas para que se obtenha posicionamento acurado. No presente artigo é proposta a definição de um novo limiar de aceitação de ocorrência de perda de ciclos, baseado nos valores de média e desvio padrão das séries de dados de triplas diferenças (TD) quando usado o método de detecção e correção de perda de ciclos baseada nas TD. Foram realizados experimentos com dados simulados em linhas de base curta e longa. Para linha de base curta, o método TD clássico e o método TD proposto apresentaram resultados similares, com sucesso de detecção em torno de 100\%. Para linha de base longa, foi obtido 31,37\% e 63,79\% de acerto na detecção, resultando em $49,45 \%$ e $95,32 \%$ de acerto na correção de perda de ciclos quando utilizado o método TD clássico e o método TD proposto. Estes resultados indicam que o método proposto se mostra relevante no contexto de detecção e correção de perda de ciclos, principalmente para linhas base longas, como é o caso no Brasil.

Palavras-chave: Perda de ciclos. Posicionamento relativo. Tripla frequência GPS.

Abstract: Carrier phase and code observable are used in high accuracy positioning by global navigation satellite systems. However, the carrier phase measurement may suffer cycle slip during signal tracking. Cycles slip is a discontinuity of an integer number of cycles in the phase observable, caused by a temporary loss of lock in the receiver carrier tracking loop. Thus, cycles slip must be detected and corrected in order to obtain accurate positioning. In the present article, is proposed the definition of a new threshold of accept the cycle slip occurrence based on the values of mean and standard deviation of triple difference (TD) series data when using the detection and correction TD method. The experiments were performed with simulated data for short baseline and long baseline. For short baseline data, the classical TD method and the proposed TD method presented similar results, around $100 \%$ accuracy in the detection. For the long baseline data, $31.37 \%$ and $63.79 \%$ of detection accuracy were obtained, resulting in $49.45 \%$ and $95.32 \%$ accuracy in the correction of cycles slip when using the classical TD method and the proposed TD method. These results indicate that the proposed method is relevant in the context of detection and correction of cycle slip, especially for long baselines, as is the case in Brazil.

Keywords: Cycle Slip. Relative positioning. GPS Triple-frequency.

\section{INTRODUÇÃO}

A observável fase da onda portadora, juntamente com a pseudodistância, são empregadas em posicionamento de alta acurácia (da ordem do centímetro ou melhor), como no posicionamento cinemático em 
tempo real (RTK - Real Time Kinematic) e posicionamento por ponto preciso, uma vez que a fase é muito mais precisa que a pseudodistância (SEEBER, 2003; MONICO, 2008). Entretanto, a medida de fase pode sofrer perda de ciclos durante o rastreio do sinal, dificultando assim a solução do vetor das ambiguidades (SEEBER, 2003).

Uma perda de ciclos é caracterizada como uma descontinuidade no número inteiro de ciclos na fase da onda portadora, causada por uma perda temporária do sinal durante o rastreamento. Tal descontinuidade pode ser causada por diversos fatores, como obstruções físicas, cintilação ionosférica, entre outros. De acordo com Seeber (2003) a amplitude pode variar de um a milhões de ciclos.

A ocorrência de perda de ciclos provoca uma mudança (salto) repentina no número inteiro de ciclos (SEBEER, 2003), alterando assim o valor da ambiguidade estimada. Logo, se torna necessário solucioná-las novamente ou corrigir a perda de ciclos. Neste sentido, para o posicionamento GPS (Global Positioning System), assim como para os demais sistemas que compõem o GNSS (Global Navigation Sattelite System), de alta acurácia, torna-se necessário que as perdas de ciclos sejam detectadas e corrigidas, quando possível. A detecção visa a identificação da ocorrência de perda de ciclos, bem como as observáveis afetadas, enquanto a correção visa determinar seu valor e corrigir as observáveis afetadas (SEBEER, 2003; HOFMANNWELLENHOF; LICHTENEGGER; WASLE, 2008).

Tendo em vista a importância da detecção e correção de perda de ciclos, diversos algoritmos foram desenvolvidos e implementados nos últimos anos para a detecção e correção de perda de ciclos para dados de dupla frequência GPS. Entretanto, com a atual modernização da constelação GPS, uma nova frequência foi adicionada, a L5. Desta forma, é necessária a otimização dos métodos para detecção e correção das perdas com dados de fase da onda portadora para tripla frequência.

Dentre os métodos para detecção e correção de perda de ciclos para tripla frequência, tem-se a tripla diferença (TD) de dados de fase da onda portadora, empregada por Monico, Souza e Machado (2009). Este é um método clássico (SEEBER, 2003) que faz uso das observáveis básicas do posicionamento relativo (Dupla Diferença - DD), cuja limiar de aceitação da ocorrência de perda de ciclos é dada em função da tetra diferença (TT). Uma das vantagens desse método é que pode ser aplicado para simples, dupla ou tripla frequência. No entanto, conforme Bisnath (2000), uma limitação desse tipo de método é a definição dos valores específicos do limiar de aceitação para a detecção. Portanto, visando sanar essa limitação, nesse artigo é apresentada uma metodologia para a melhoria do limiar de aceitação da ocorrência de perda de ciclos, baseado nos valores de média e desvio padrão das séries de dados de triplas diferenças (TD).

\section{MÉTODOS DE DETECÇÃo E CORREÇÃO DE PERDA DE CICLOS}

Conforme Monico, Souza e Machado (2009), a parte fracionária da fase não é afetada na ocorrência de uma perda de ciclos, apenas o número inteiro. Assim, enquanto o rastreamento ocorre de forma contínua, a ambiguidade $N_{r_{0}}^{s}$ permanece a mesma até o momento da ocorrência de uma perda de ciclos na época $(t)$, sendo este alterado então para $N_{r_{1}}^{s}$ a partir da época $(t+d t)$.

As perdas de ciclos podem ocorrer de forma independente em cada uma das três frequências e em cada satélite GPS e afetam todas as observações subsequentes à sua ocorrência (DAI, KNEDLIK, LOFFELD, 2009). Neste sentido, faz-se necessária a detecção e correção de perda de ciclos, quando possível, na observável de fase para a realização do posicionamento GPS de alta acurácia. Tendo em vista o cenário atual, com a modernização do GPS, diversos estudos vêm sendo realizados de forma a avaliar os impactos e benefícios de adoção do sinal na terceira frequência na detecção e correção de perda de ciclos, como em Monico, Souza e Machado (2009), Dai, Knedlik e Loffeld (2009), Lacy, Reguzzoni e Sansò (2012), Chen et al. (2015), Zhang e Li (2015), Teunissen e Bakker (2015), Gu e Zhu (2017), entre outros.

Para a detecção e correção, uma vez que as perdas de ciclos são eventos aleatórios, os algoritmos de detecção de perda de ciclos devem ser aplicados época a época, rápidos e de baixo custo computacional (DAI; KNEDLIK; LOFFELD, 2009). Visando a detecção e correção de perda de ciclos em dados de tripla frequência GPS, será discutido na sequência o método baseado nas triplas (TD) e tetra (TT) diferenças, apresentado por Monico, Souza e Machado (2009). A escolha deste método se deu levando em consideração a aplicabilidade 
de dados de tripla-frequência do GPS modernizado, facilidade de implementação, além de apresentarem resultados promissores em Monico, Souza e Machado (2009).

\subsection{Método das triplas diferenças}

Considerando a equação de dupla diferença de fase, uma TD é caracterizada como a diferença de duas DD entre duas épocas adjacentes, envolvendo os mesmos satélites e receptores (SEEBER, 2003). Na ausência de perda de ciclos, a série temporal das medidas de fase da onda portadora, bem como suas diferenciações, devem ser contínuas. Assim, um salto abrupto na curva da TD é um forte indicativo da ocorrência de perda de ciclos (MONICO; SOUZA; MACHADO, 2009).

Se uma perda de ciclos na observação de fase na estação $i$ e satélite $s$ ocorreu em uma determinada época $t$, todas as DD deste conjunto de observações a partir da época $t$ serão afetados. Entretanto, somente a TD na época $t$ que envolve a estação $i$ e o satélite $s$ é afetada pela perda de ciclos. A Figura 1 apresenta o procedimento descrito, apontando a ocorrência de perda de ciclos na época 80 . O salto na série temporal da TD indica a ocorrência de perda de ciclos.

Uma tetra diferença (TT) é caracterizada como a diferenças entre duas TD em épocas adjacentes, envolvendo assim três épocas de dados (MONICO; SOUZA; MACHADO, 2009). Para detecção das perdas de ciclos, ou seja, identificar a ocorrência de saltos, são empregadas TT, também ilustrada na Figura 1. Um salto na TT, além de ser um indicativo para detecção da perda de ciclos, pois tais descontinuidades ficam mais acentuadas, proporcionam um valor aproximado para o número de ciclos dessa perda (MONICO; SOUZA; MACHADO, 2009).

Figura 1 - Dupla, tripla e tetra diferença da observação de fase.
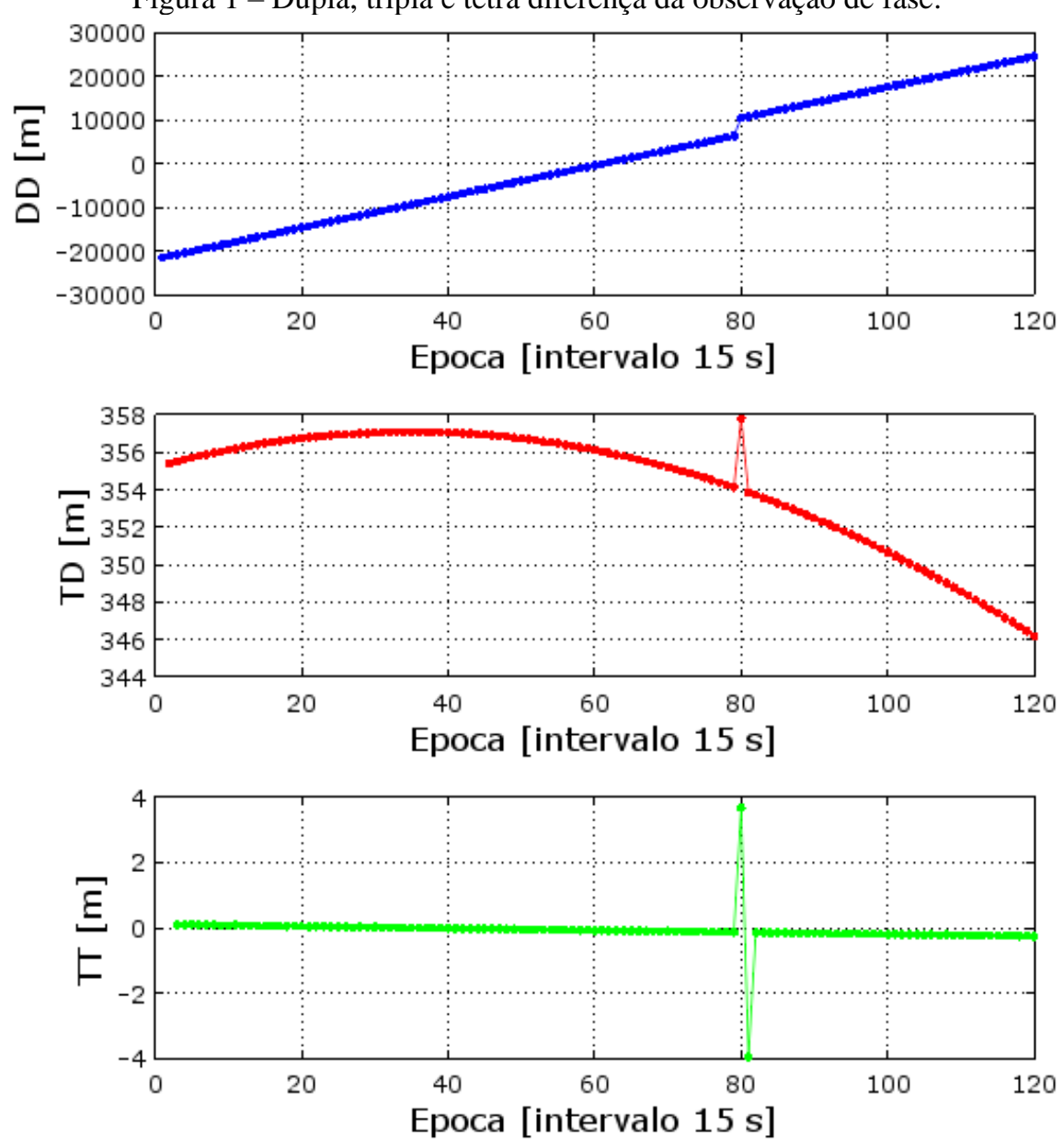

Fonte: Os autores (2021).

O limiar para aceitar a detecção da ocorrência de uma perda de ciclos, de acordo com o proposto em Monico, Souza e Machado (2009), é dado em função do comprimento da linha de base empregada no 
posicionamento relativo, comparando valores específicos com o valor dos saltos presentes nas séries de TT. Contudo, conforme Bisnath (2000), a principal desvantagem desse tipo de método está relacionada a definição dos valores específicos do limiar de aceitação na deteç̧ão.

Uma vez que uma ou mais perdas tenham sido detectadas, uma estratégia adotada para a correção envolve a alteração dos elementos da Matriz de Variância-Covariância (MVC) dos parâmetros envolvidos com a ocorrência da perda de ciclos (MONICO; SOUZA; MACHADO, 2009). Neste sentido, para realizar a correção, deve-se atribuir um grande valor de variância na MVC para a DD da ambiguidade que envolve o satélite em que ocorreu a perda de ciclos. Segundo Monico, Souza e Machado (2009), para realizar a alteração da MVC, é adotado como valor de desvio padrão da observável o valor aproximado da perda de ciclos obtido na TT escalonado por três. Além disto, as correlações com os outros parâmetros podem ser negligenciadas, ou seja, tomadas como nulas. Esta abordagem pode ser aplicada em cada uma das três frequências.

\section{METODOLOGIA PARA MELHORIA DO LIMIAR PARA O MÉTODO DAS TRIPLAS DIFERENÇAS}

Para linhas de base longa o método das TD original proposto por Monico, Souza e Machado (2009), cujo limiar de aceitação utiliza-se das TT, não apresentou grande eficácia na detecção de perdas de poucos ciclos em linhas de base longa, conforme verificado em Mendonça (2019). Assim, foram realizados estudos visando a reavaliação do limiar de aceitação de ocorrência de perda de ciclos para o método. Foi proposto um novo limiar levando em consideração os valores de média e desvio padrão da série de dados de TD, conforme a ideia proposta por Blewitt (1990).

Por meio das observáveis de TD, as quais são calculadas época por época em cada frequência e assumindo-se um desvio padrão a priori $\left(\mathrm{s}_{0}\right)$, o algoritmo atualiza sequencialmente o valor de $\overline{T D}$, média dos valores de TD em cada frequência, e seu desvio padrão $\left(s_{T D}\right)$ pelas Eq. (1) e (2), respectivamente, adaptado de Blewitt (1990):

$$
\begin{gathered}
\overline{T D}_{i}=\overline{T D}_{i-1}+\frac{1}{i}\left(\mathrm{TD}_{i}-\overline{T D}_{i-1}\right), \\
s_{T D_{\mathrm{i}}}^{2}=s_{T D_{\mathrm{i}-1}^{2}}^{2}+\frac{1}{i}\left[\left(\mathrm{TD}_{i}-\overline{T D}_{i-1}\right)^{2}-s_{T D_{\mathrm{i}-1}^{2}}^{2}\right],
\end{gathered}
$$

sendo $i$ o número atual de épocas em um conjunto de dados sem perda de ciclos.

Para utilização desta metodologia, tornou-se necessária a determinação do valor de desvio padrão $a$ priori $\left(\mathrm{s}_{0}\right)$, o qual é influenciado pelo comprimento da linha de base. Neste sentido, foram selecionadas estações da Rede Brasileira de Monitoramento Contínuo dos Sistemas GNSS (RBMC) formando diferentes comprimentos de linhas de base, a fim de estabelecer o valor de $s_{0}$ adequado. Para cada linha de base selecionada foi considerado um conjunto de dados com 50 épocas, com intervalo amostral 15 segundos, para o dia 20 de julho de 2018. Os dados da amostra passaram, inicialmente, por verificação por outros métodos de detecção, a fim de garantir a não ocorrência de perda de ciclos e desta forma, validar os resultados obtidos.

Na sequência, foram simuladas perda de ciclos em uma estação do par, sendo a outra tomada como base. Iniciando na época 3 com a inserção de uma perda de 1 ciclo, foi inserida a cada 3 épocas uma perda de ciclos incrementada por 1 ciclo para as três frequências (L1, L2 e L5). Em seguida, foram testados diferentes valores de $\mathrm{s}_{0}$ até determinar qual valor proporcionaria maior porcentagem de acerto na detecção de perda com menor número de ciclos possível, evitando as falsas detecções. Este procedimento foi repetido para os 10 pares de estações selecionados, sendo os resultados obtidos apresentados na Tabela 1. 
Tabela 1 - Valor do desvio padrão a priori obtido em função do comprimento da linha de base.

\begin{tabular}{c|c|c}
\hline Linha de base & Comprimento $\mathbf{K m})$ & $\mathbf{s}_{\mathbf{0}}$ \\
\hline PPTE - PRU4 & 0,020 & 0,5 \\
\hline RIOD - ONRJ & 12,044 & 0,7 \\
\hline POLI - EACH & 24,944 & 1,0 \\
\hline SPC1 - SPPI & 58,963 & 1,5 \\
\hline PPTE - SPDR & 74,438 & 1,9 \\
\hline SPAR - SPTU & 82,614 & 2,0 \\
\hline PPTE - SPTU & 96,925 & 2,1 \\
\hline SPAR - SPDR & 119,623 & 2,3 \\
\hline SPDR - SPTU & 121,474 & 2,3 \\
\hline PPTE - SPAR & 144,155 & 2,5 \\
\hline
\end{tabular}

Fonte: Os autores (2021).

Desta maneira foi possível determinar o valor de $\mathrm{s}_{0}$. Definido o valor de $\mathrm{s}_{0}$ para o conjunto de dados apresentados na Tabela 1, foi ajustada uma função polinomial $\left(P(x)=a_{0}+a_{1} x+\cdots+a_{n} x^{n}\right)$ para determinar o valor de $s_{0}$ em comprimentos de linha de base distintos. Para tanto, utilizou-se um polinômio de grau 5, uma vez que esta foi a função que melhor se ajustou aos dados empregados. A Figura 2 representa a função $P(x)$ ajustada com base nos valores de $\mathrm{s}_{0}$ estabelecidos.

Figura 2 - Função $P(x)$ para os valores de $\mathrm{s}_{0}$.

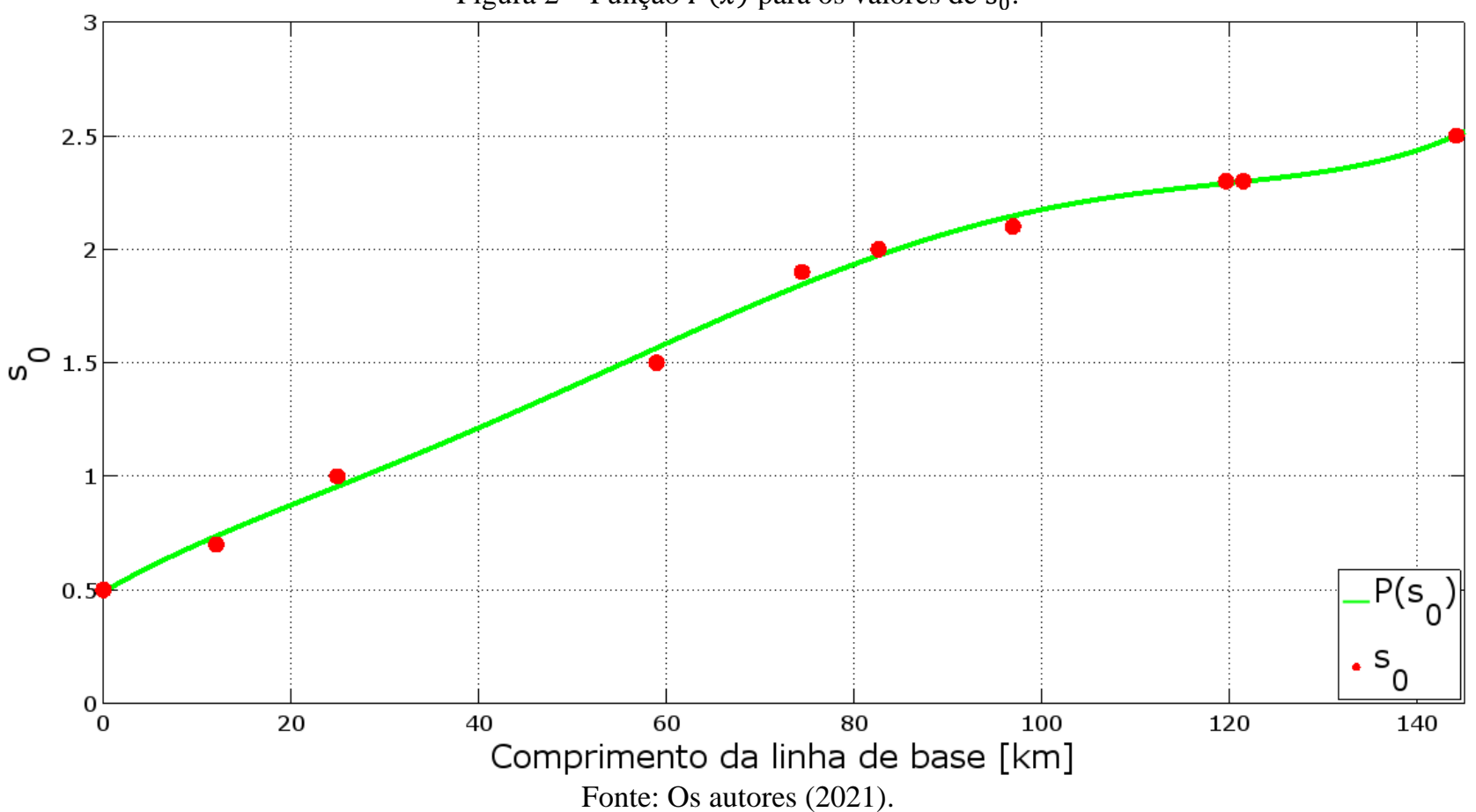

Uma vez estabelecido o valor de $\mathrm{s}_{0}$, as Eq. (1) e (2) podem ser aplicadas para calcular sequencialmente os valores de média e desvio padrão da série de TD.

A fim de determinar se uma perda de ciclos na série de TD ocorreu, deve-se testar se houve um salto no bias $\left(b_{T D}\right)$ da série. Assumindo distribuição gaussiana (WOLF e GHILANI, 1997), é possível estabelecer o seguinte intervalo de confiança de $95 \%$, nível de significância $\alpha=0,05$ :

$$
P\left(b_{T D_{i-1}}-1,96 \frac{s_{T D_{i}}}{\sqrt{i}}<b_{T D_{i}}<b_{T D_{i-1}}+1,96 \frac{s_{T D_{i}}}{\sqrt{i}}\right)
$$

em que $b_{T D_{i}}=\mathrm{TD}_{i}-\overline{T D}_{i-1}$ e $b_{T D_{i-1}}=\mathrm{TD}_{i-1}-\overline{T D}_{i-2}$. Na inicialização dos cálculos, toma-se $b_{T D_{i-1}}=$ $b_{T D_{i}}$. Caso o valor de $b_{T D_{i}}$ se encontre fora do intervalo de confiança estabelecido, assume-se que uma perda 
de ciclos ocorreu e um novo cálculo da média é iniciado até a ocorrência de outra perda de ciclos.

\section{EXPERIMENTOS, RESULTADOS E ANÁLISES}

Para verificar a eficácia do limiar proposto para o método das TD, foram realizados dois experimentos com dados simulados: o primeiro para linha de base curta e o segundo para linha de base longa. Na condução dos mesmos, foi considerado período de baixa atividade ionosférica, sendo os dados coletados no dia 20 de julho de 2018.

As perdas de ciclos foram inseridas (somadas) nos dados originais em diferentes épocas, variando de poucos a muitos ciclos (1 a 20 ciclos). As perdas de ciclos foram simuladas de forma que ocorressem perdas com mesmo número de ciclos em mais de uma frequência simultaneamente, conforme Tabela 2, nos dois experimentos.

Tabela 2 - Perda de ciclos simuladas.

\begin{tabular}{|c|c|c|c|}
\hline \multirow{2}{*}{ Época } & \multicolumn{3}{|c|}{ Número de ciclos } \\
\hline & L1 & L2 & L5 \\
\hline 10 & 5 & 3 & 0 \\
\hline 20 & 2 & 4 & 7 \\
\hline 30 & 0 & 1 & 1 \\
\hline 40 & 0 & 17 & 0 \\
\hline 50 & 1 & 0 & 0 \\
\hline 52 & 2 & 1 & 1 \\
\hline 55 & 1 & 2 & 2 \\
\hline 57 & 4 & 3 & 3 \\
\hline 60 & 7 & 5 & 5 \\
\hline 70 & 1 & 1 & 1 \\
\hline 80 & 20 & 16 & 14 \\
\hline 90 & 4 & 0 & 0 \\
\hline 92 & 8 & 7 & 6 \\
\hline 95 & 11 & 5 & 12 \\
\hline 97 & 17 & 14 & 10 \\
\hline 100 & 2 & 10 & 19 \\
\hline 110 & 8 & 8 & 8 \\
\hline
\end{tabular}

Fonte: Os autores (2021).

No que concerne a detecção de perda de ciclos para dados GPS de tripla frequência, foi testado o método das TD com o limiar original (baseado na TT) e com o limiar proposto (baseado no critério estatístico). Uma vez realizada a detecção das perdas de ciclos, o próximo passo foi realizar a correção destas utilizado o valor obtido na TT para relaxar a MVC dos parâmetros durante o ajustamento.

Para avaliar o impacto da ocorrência de perda de ciclos, bem como a influência da correção foi realizado o posicionamento relativo, utilizando a versão inicial do software FCT-RTK-Net, um sistema científico que vem sendo desenvolvido na FCT/UNESP (SILVA, 2015). O software utilizado faz uso da estimativa de mínimos quadrados recursiva (Filtragem de Kalman), permitindo atualizar a solução do ajustamento com a introdução de novas observações. A solução do posicionamento relativo deu-se empregando as observáveis de fase e pseudodistância para as observações de dupla e tripla frequência, quando disponíveis. Foram consideradas a utilização de efemérides precisas e máscara de elevação de $10^{\circ}$.

Uma vez realizado o posicionamento relativo, foram determinadas as coordenadas cartesianas $(\mathrm{X}, \mathrm{Y}$, Z) e respectivos desvios-padrão $\left(\sigma_{X}, \sigma_{Y}, \sigma_{Z}\right)$ para a estação de interesse época a época, as quais foram comparadas com as coordenadas de referência, de forma a obter o erro no posicionamento. Para facilitar as análises, o erro foi transformado para um Sistema de Coordenadas Local (STL), permitindo avaliar os resultados em termos de componente horizontal e vertical. Na sequência, foi calculada uma medida de acurácia denominada raiz quadrada do erro quadrático médio (REMQ), dada por (MONICO et. al, 2009): 


$$
R E M Q=\sqrt{s^{2}+t^{2}}
$$

sendo $s^{2}$ a dispersão das medidas (variância) e $t$ a tendência ou vício do estimador (discrepância em relação ao valor verdadeiro). Ademais, foi considerado o valor da média do REMQ e seu respectivo desvio padrão.

\subsection{Linha de base curta}

Foram utilizados dados de uma linha de base curta, com distância aproximada de 263,3 m, considerando as estações PRU4 e PRU2, ambas pertencentes a rede CIGALA/CALIBRA, localizadas nas dependências da Universidade Estadual Paulista (Unesp), Faculdade de Ciências e Tecnologia, Presidente Prudente - SP, como pode ser visto na Figura 3.

Figura 3 - Localização das estações da rede CIGALA/CALIBRA.

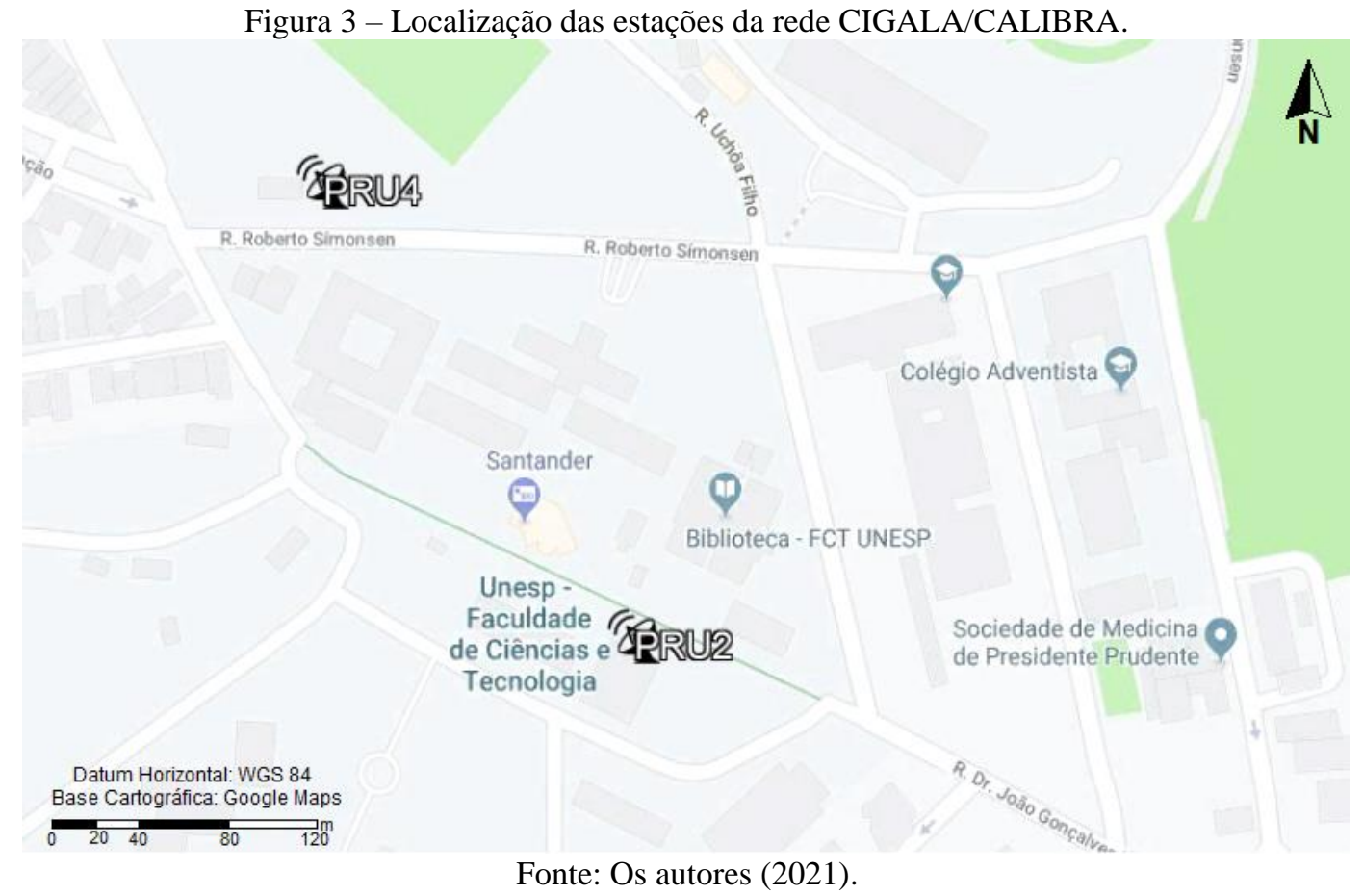

Estudos nestas condições são importantes pois os erros devido a ionosfera e troposfera podem ser considerados nulos no posicionamento relativo. Os dados de observação empregados formam uma amostra de 30 minutos, com intervalo amostral entre épocas de 15 segundos, com início as $7 \mathrm{~h}$ TU. Inicialmente foi realizado o posicionamento relativo com dados sem perda de ciclo, tomando como base a estação PRU4, permitindo assim a determinação das coordenadas da estação PRU2 com acurácia posicional tridimensional REMQ de 0,206 $\pm 0,013 \mathrm{~m}$.

Na sequência foram simuladas perdas de ciclos nas observáveis de fase coletadas na estação PRU2 para o satélite GPS G26, que transmite dados de tripla frequência de acordo com a Tabela 2. Realizando o posicionamento novamente, obteve-se que a acurácia tridimensional de 47,954 $\pm 2,945 \mathrm{~m}$, comprovando que perda de ciclos degradam consideravelmente o posicionamento GNSS.

Para aplicar método de detecção e correção de perda de ciclos, foram calculadas as TD considerando o par de estações PRU4-PRU2. Os valores das séries de TD para o satélite G26 nas três frequências são apresentados na Figura 4. 
Figura 4 - Séries de TD do satélite G26 para L1, L2 e L5.
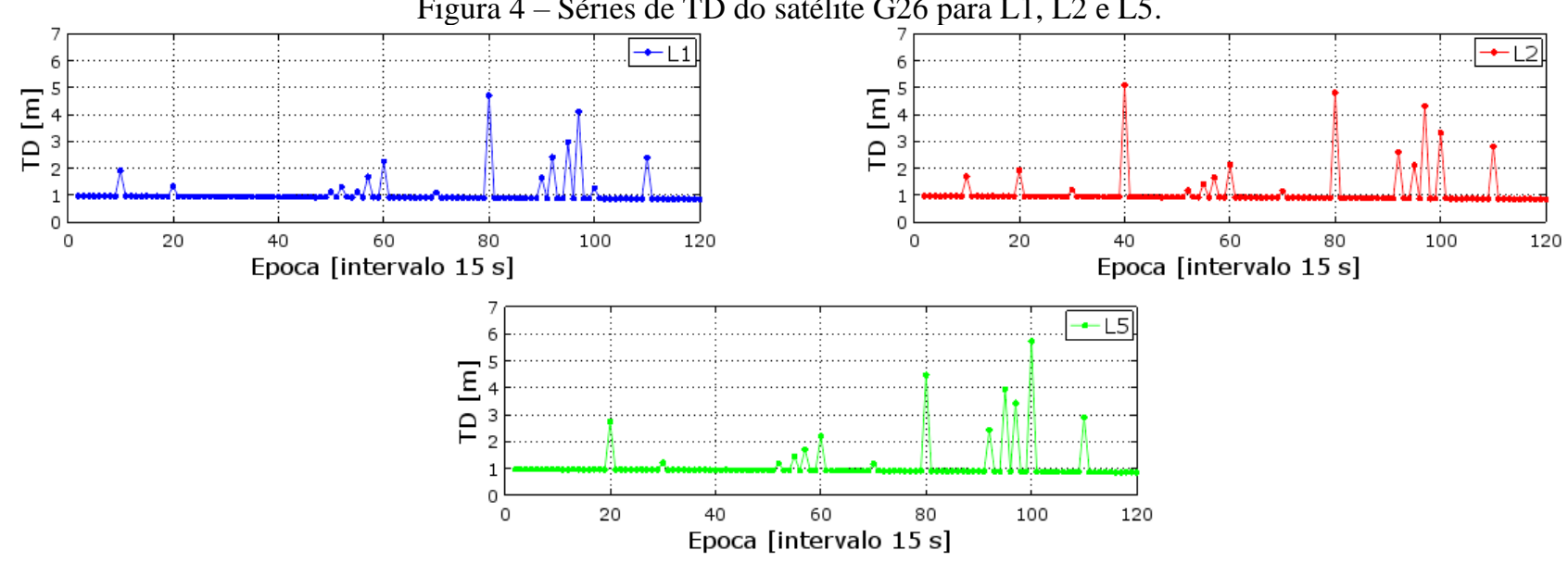

Fonte: Os autores (2021).

Como observado na Figura 4, são encontradas descontinuidades evidentes nas séries de TD para as três frequências, as quais são forte indicativo da ocorrência de perda de ciclos. Nota-se que os saltos são proporcionais as perdas simuladas (Tabela 2), quanto maior o salto, maior o número de ciclos na perda.

Nesse contexto, o uso do limiar original, baseado nas séries de TT e no comprimento da linha de base, apresentou bons resultados. Com este limiar, o método foi capaz de detectar corretamente todas as perdas de ciclos inseridas, tanto aquelas de muitos ciclos, quanto as de 1 ciclo. Deste modo, o limiar original apresentou $100 \%$ de acerto na detecção de perda de ciclos em uma linha de base curta. Ressalta-se também que foram detectadas corretamente as perdas que ocorreram sequencialmente, tanto para aquelas de poucos ciclos, entre as épocas 50 e 60, quanto para aquela de muitos ciclos, entre as épocas 90 e 100.

$\mathrm{Na}$ sequência, foi considerada a metodologia proposta para melhoria do limiar de aceitação da ocorrência de perdas, dado em função do intervalo de confiança dos valores de média da série de dados de TD. O uso do limiar proposto apresentou índice de $90,91 \%$ de acerto na detecção de perda de ciclos para uma linha de base curta. Este índice indica que as perdas de ciclos inseridas foram detectadas pelo limiar proposto em quase sua totalidade, com exceção a uma perda não detectada e 4 falsas detecções na ocorrência de perdas de poucos ciclos em épocas subsequentes, entre as épocas 50 e 60. Destaca-se que a utilização do limiar proposto, considerando uma linha de base curta, também foi capaz de detectar tanto as de muitos ciclos, quanto aquelas de 1 ciclo. Ademais, foram detectadas corretamente as perdas de muitos ciclos que ocorrem entre as épocas 90 e 100 consecutivamente, bem como as perdas de poucos ciclos que ocorreram sequencialmente entre as épocas 50 e 60, com ressalva feita as falsas detecções em L1.

Na realização da correção de perda de ciclos, o valor aproximado das TT foi utilizado para relaxar a MVC dos parâmetros durante o ajustamento para ambas metodologias de detecção avaliadas. Aplicando este procedimento e realizando o posicionamento relativo, foram estimadas as coordenadas da estação PRU2, bem como o erro no posicionamento no STL e respectivo desvio padrão em cada época, permitindo assim a determinação da acurácia posicional pela REMQ.

Comparando os resultados obtidos sem correção de perda de ciclos com os obtidos após a correção, foi possível constatar que: houve melhoria na estimativa tridimensional das coordenadas da estação PRU2 de 99,91\% no que concerne aos efeitos de perda de ciclos detectadas pelo limiar original, atingindo acurácia tridimensional $0,249 \pm 0,012 \mathrm{~m}$; enquanto que houve melhoria de $99,88 \%$ na estimativa tridimensional ao detectar as perdas e ciclos pelo limiar proposto, correspondendo a uma acurácia tridimensional 0,263 $\pm 0,011$ m.

Os resultados obtidos para dados simulados para linha de base curta podem ser sintetizados pela Tabela 3. 
Tabela 3 - Síntese dos resultados obtidos com dados simulados em linha de base curta.

\begin{tabular}{c|c|c|c}
\hline Método utilizado & $\begin{array}{c}\text { Acerto na } \\
\text { detecção }\end{array}$ & $\begin{array}{c}\text { REMQ médio } \\
\text { tridimensional (m) }\end{array}$ & $\begin{array}{c}\text { Melhoria com } \\
\text { correção }\end{array}$ \\
\hline Sem perda de ciclos & -- & 0,206 & -- \\
\hline Perda de ciclos simuladas & -- & 47,954 & -- \\
\hline Triplas Diferenças com limiar baseado na TT & $100,00 \%$ & 0,249 & $99,91 \%$ \\
\hline $\begin{array}{c}\text { Triplas Diferenças com limiar baseado em critério } \\
\text { estatístico na TD }\end{array}$ & $90,91 \%$ & 0,263 & $99,88 \%$ \\
\hline
\end{tabular}

Fonte: Os autores (2021).

\subsection{Linha de base longa}

Foi considerada uma linha de base longa, com distância aproximada de 82,6 km, formada pelas estações SPAR e SPTU, ambas pertencentes a RBMC, localizadas em Araçatuba - SP e Tupã - SP, respectivamente, como pode ser visto na Figura 5.

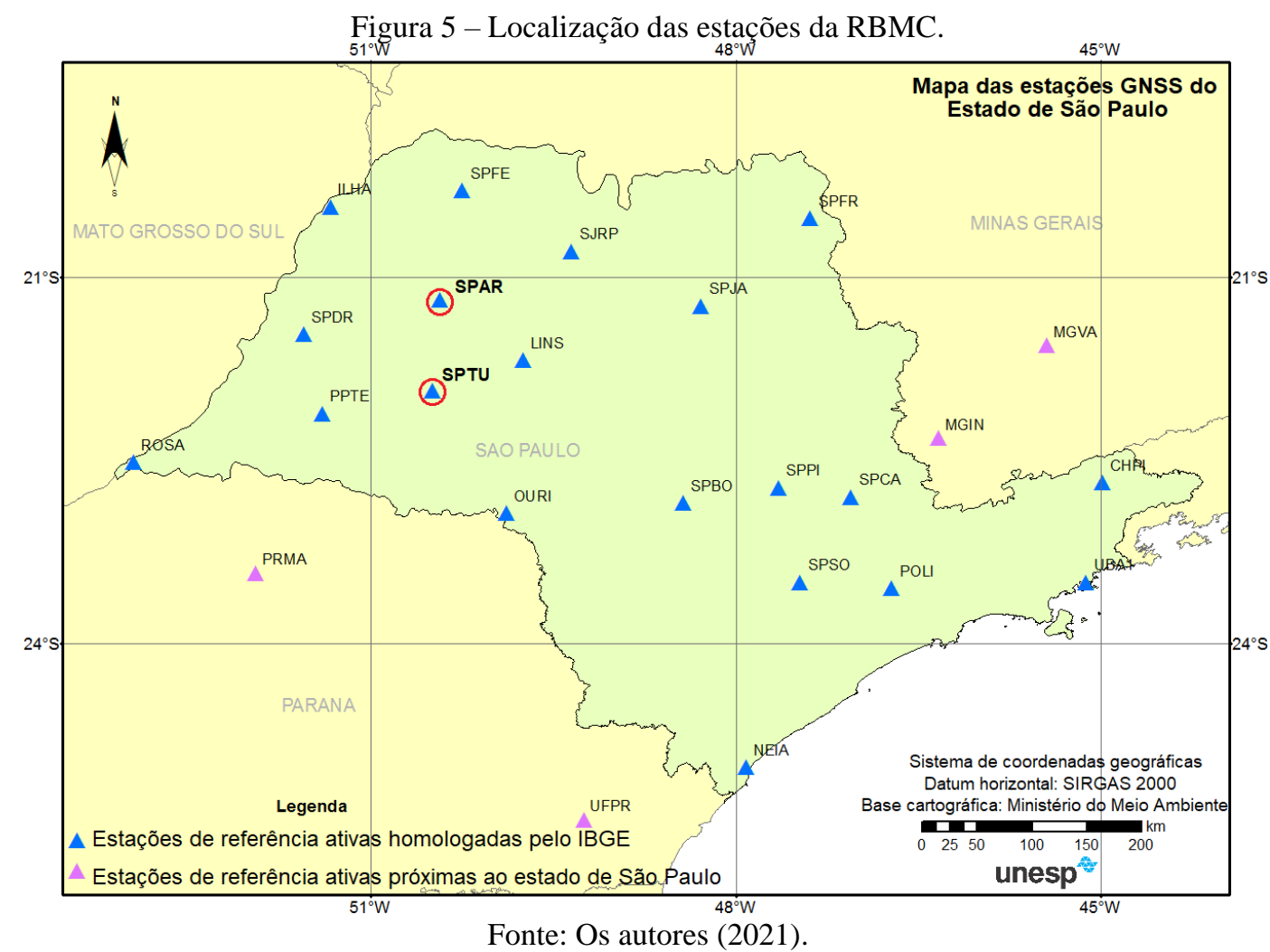

Os dados de observação empregados formam uma amostra de 30 minutos de observação, com intervalo amostral entre épocas de 15 segundos, sendo este coletado no dia 20 de julho de 2018, tendo início as 06h TU. Inicialmente foi realizado o posicionamento relativo, tomando como base a estação SPAR, permitindo assim a determinação das coordenadas da estação SPTU com acurácia posicional tridimensional pela REMQ de 0,631 $\pm 0,029 \mathrm{~m}$.

Na sequência foram simuladas perdas de ciclos nas observáveis de fase coletadas na estação SPTU para o satélite GPS G26, que transmite dados de tripla frequência de acordo com a Tabela 2. Realizando o posicionamento novamente, obteve-se que a acurácia tridimensional de 29,567 $\pm 1,706 \mathrm{~m}$, comprovando novamente que perda de ciclos degradam o posicionamento GNSS.

Para utilizar o método das triplas diferenças, foram calculadas as séries de TD considerando o par de estações SPAR-SPTU, sendo os valores das séries para o satélite G26 nas três frequências apresentados na Figura 6. 
Figura 6 - Séries de TD do satélite G26 para L1, L2 e L5.
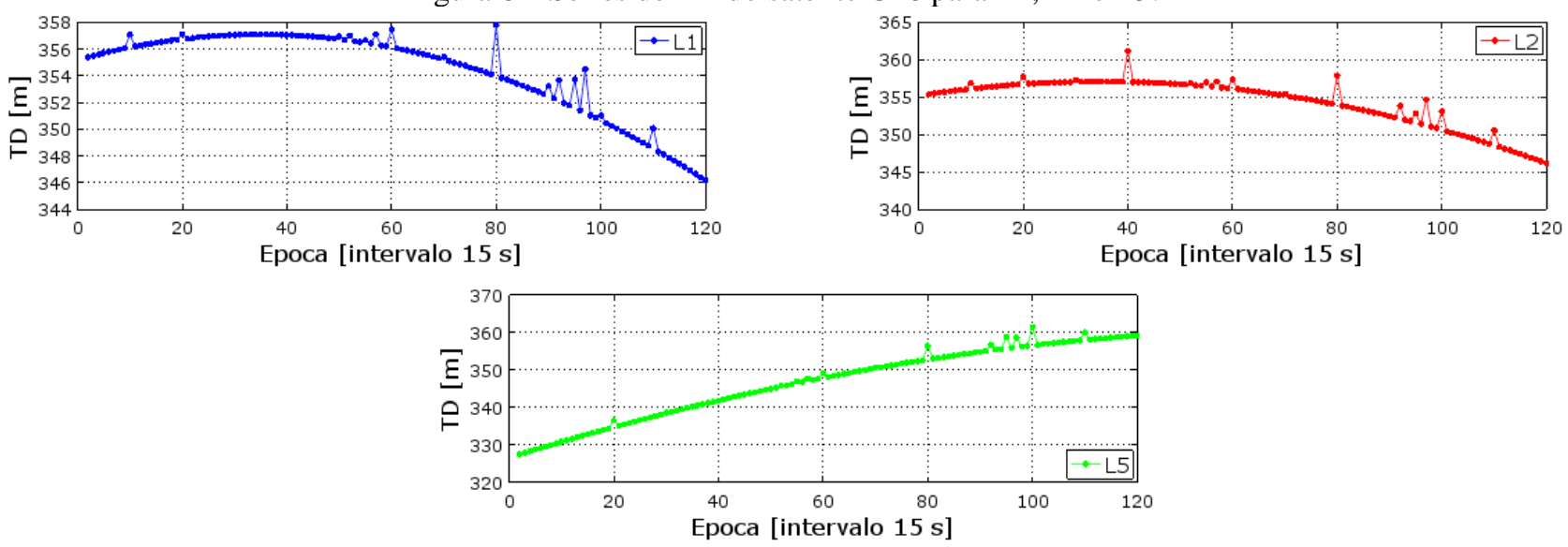

Fonte: Os autores (2021).

Na Figura 6, foram encontradas descontinuidades nas séries de TD nas três frequências, as quais são indicativos da ocorrência de perda de ciclos. Diferentemente do ocorrido na linha de base curta, tem-se que os saltos nas séries de TD para uma linha de base longa são mais sutis, o que dificulta a detecção de perda de ciclos.

Considerando o uso do limiar original em função das séries de TT, o método das triplas diferenças não se mostrou eficaz em linha de base longa. Por este limiar, foram detectadas somente as perdas com valor maior ou igual a 12 ciclos, as quais equivalem aos maiores picos das séries de TD (Figura 6). De modo geral, a detecção de perda de ciclos levando em consideração o limiar original apresentou índice de 31,37\% de acerto em uma linha de base longa. Tal índice representa 16 acertos na detecção, não sendo este um resultado satisfatório.

Considerou-se então a metodologia proposta para melhoria do limiar de aceitação da ocorrência de perda de ciclos. O limiar definido em função de critérios estatísticos para as séries de TD foi capaz de detectar corretamente as perdas de muitos ciclos em sua grande maioria, bem como algumas das perdas de poucos ciclos. Constatou-se também que parte das perdas de ciclos não foram detectadas, totalizando 14 perdas de ciclos não detectadas. As não detecções pelo limiar proposto se deram na ocorrência de perdas de poucos ciclos, principalmente em perdas consecutivas entre as épocas 50 e 60. Ademais, foram encontradas 7 falsas detecções, as quais se deram na ocorrência de perdas em épocas subsequentes. De modo geral, o limiar proposto apresentou índice de 63,79\% de acerto na detecção de perda de ciclos, o que corresponde a uma melhoria de 2 vezes no que se refere a detecção quando comparado ao limiar original.

Assim como para linha de base curta, para a realização da correção de perda de ciclos o valor aproximado das TT foi utilizado para relaxar a MVC dos parâmetros durante o ajustamento para ambas metodologias de detecção avaliadas. Aplicando este procedimento e realizando o posicionamento relativo, foram estimadas as coordenadas da estação SPTU, bem como o erro no posicionamento no STL e respectivo desvio padrão em cada época, permitindo assim a determinação da acurácia posicional pela REMQ.

Comparando os resultados obtidos sem correção de perda de ciclos com os obtidos após a correção, foi possível constatar que: ao fazer uso do limiar original na detecção houve uma melhoria na estimativa tridimensional das coordenadas da estação SPTU de 49,45\% no que concerne aos efeitos da perda de ciclos, atingindo acurácia tridimensional 15,257 $\pm 0,469 \mathrm{~m}$; enquanto que, ao utilizar o limiar proposto em função de critérios estatísticos houve melhoria de $95,32 \%$ na estimativa tridimensional no que concerne aos efeitos da perda de ciclos, correspondendo a uma acurácia tridimensional 1,984 $\pm 0,113 \mathrm{~m}$.

Comparando os resultados obtidos para os dados originais com os obtidos após a correção ser aplicada constatou-se que: houve uma piora de 24 vezes na estimativa tridimensional quando utilizado o limiar de detecção original; e uma piora 3 vezes na estimativa tridimensional quando utilizado o limiar de detecção proposto. Considerando o valor da média da REMQ tridimensional após utilização do limiar de detecção proposto, tem-se que este foi da ordem de $2,0 \mathrm{~m}$, sendo este um resultado não esperado ao posicionamento relativo. Contudo, levando em conta que a média da REMQ tridimensional obtido com utilização do limiar de 
detecção original foi da ordem de $15 \mathrm{~m}$, ficou evidente que o estabelecimento de um novo critério para aceitação da ocorrência de perda de ciclos trouxe grande avanço para utilização do método das triplas diferenças em linhas de base longa.

Os resultados obtidos para dados simulados para linha de base curta podem ser sintetizados pela Tabela 4.

Tabela 4 - Síntese dos resultados obtidos com dados simulados em linha de base longa.

\begin{tabular}{c|c|c|c}
\hline Método utilizado & $\begin{array}{c}\text { Acerto na } \\
\text { detecção }\end{array}$ & $\begin{array}{c}\text { REMQ médio } \\
\text { tridimensional (m) }\end{array}$ & $\begin{array}{c}\text { Melhoria com } \\
\text { correção }\end{array}$ \\
\hline Sem perdas de ciclo & -- & 0,631 & -- \\
\hline Perda de ciclos simuladas & -- & 29,567 & -- \\
\hline $\begin{array}{c}\text { Triplas Diferenças com limiar baseado em critério } \\
\text { estatístico na TD }\end{array}$ & $31,37 \%$ & 15,265 & $49,45 \%$ \\
\hline
\end{tabular}

Fonte: Os autores (2021).

\section{CONCLUSÃO}

Nos experimentos realizados foram empregados dados simulados, necessários para permitir a análise dos resultados da detecção considerando situações diversas e controladas, onde ficou evidente por meio dos resultados que a ocorrência de perda de ciclos nas observáveis de fase dos satélites GPS degrada consideravelmente a qualidade do posicionamento.

Do que foi discutido no artigo, tem-se que o método das TD faz uso das observáveis básicas do posicionamento relativos (DD) e pode ser aplicado para simples, dupla ou tripla frequência do GPS modernizado. Em contra partida, o limiar de aceitação para ocorrência de perda de ciclos originalmente proposto apresentou deficiência para linha de base longas, necessitando a revisão do mesmo. Assim, foi proposto limiar de aceitação da ocorrência de perda de ciclos baseado no critério estatístico dos valores de média e desvio padrão da série de dados de TD.

Dos resultados obtidos, constatou-se que o limiar proposto apresentou resultados satisfatórios em linha de base curta, entretanto, apresentou algumas falsas detecções. Em se tratando de linha de base longa, o limiar proposto foi capaz de detectar corretamente perdas de muitos ciclos em sua grande maioria, bem como algumas perdas de poucos ciclos, com ressalvas a algumas falsas detecções. Assim sendo, ao fazer uso do limiar proposto para linha de base longa, o resultado no posicionamento se mostrou duas vezes melhor do que aquele obtido com o limiar original baseado nas TT.

Contudo, a metodologia proposta para melhoria do limiar de aceitação de ocorrência de perda de ciclos pode ser aprimorada, elevando o índice de acerto da mesma e evitando a ocorrência de falsas detecções. Recomenda-se utilizar linhas de base maiores que $150 \mathrm{~km}$ para estimativa do valor de desvio padrão a priori $\left(s_{0}\right)$. Neste aspecto, tem-se que o valor de $s_{0}$ foi estimado com dados em período de baixa atividade ionosférica, sendo aconselhado utilizar outros períodos de dados para avaliar o impacto de $s_{0}$ nessas situações. Por fim, indica-se fazer experimentos considerando dados não simulados, bem como em períodos com alta atividade ionosférica, incluindo cintilação ionosférica.

Considerando os resultados obtidos, tem-se que a metodologia para melhoria do limiar de detecção de perda de ciclos mostrou-se relevante, trazendo avanço para utilização do método das triplas diferenças, principalmente em linha de base longa. Porém mais estudos devem ser realizados para verificar a eficiência do limiar proposto e trazer aprimoramentos ao mesmo.

\section{Agradecimentos}

Ao apoio financeiro da Coordenação de Aperfeiçoamento de Pessoal de Nível Superior - Brasil (CAPES), sob forma de bolsa de demanda social durante o período de três meses. Ao apoio financeiro da Fundação de Amparo à Pesquisa do Estado de São Paulo (FAPESP), sob forma de bolsa de demanda social 
(Processo: 2016/26000-5). Ao Instituto Nacional de Ciência e Tecnologia - Tecnologia GNSS no Suporte à Navegação Aérea (INCT GNSS-NavAer), financiado pelo CNPq (Conselho Nacional de Desenvolvimento Científico e Tecnológico - processo 465648/2014-2), FAPESP (Fundação de Amparo à Pesquisa do Estado de São Paulo - processo 2017/50115-0) e CAPES (Coordenação de Aperfeiçoamento de Pessoal de Nível Superior - processo $\left.\mathrm{n}^{\circ} 88887.137186 / 2017-00\right)$.

\section{Contribuição dos Autores}

Conceptualização: D.B.M.A. Metodologia: D.B.M.A., C.H.C.M., C.M.S. Software: C.H.C.M., C.M.S. Visualização: C.H.C.M., D.B.M.A. Redação - minuta inicia: C.H.C.M., D.B.M.A. Redação - minuta final: C.H.C.M., D.B.M.A.

\section{Conflitos de Interesse}

Os autores declaram que não há algum conflito de interesse.

\section{Referências}

BISNATH, S. B. Efficient, automated cycle-slip correction of dual-frequency kinematic GPS data. In: Proceedings of the 13th International Technical Meeting of the Satellite Division of The Institute of Navigation (ION GPS 2000). Salt Lake City: Institute of Navigation, 2000. p. 145-154.

BLEWITT, G. An automatic editing algorithm for GPS data. Geophysical Research Letters, Wiley Online Library, v. 17, n. 3, p. 199-202, 1990.

CHEN, D. et al. A double-differenced cycle slip detection and repair method for GNSS cors network. GPS Solutions, Springer, p. 1-12, 2015.

DAI, Z.; KNEDLIK, S.; LOFFELD, O. Instantaneous triple-frequency GPS cycle-slip detection and repair. International Journal of Navigation and Observation, Hindawi Publishing Corporation, v. 2009, 2009.

GU, X. ZHU. B. Detection and correction of cycle slip in triple-frequency GNSS positonig. IEEE Access. v. 5, 2017. p.12584-12595.

HOFMANN-WELLENHOF, B.; LICHTENEGGER, H.; WASLE, E. GNSS - Global navigation satellite system: GPS, GLONASS, Galileo, and more. Morlenbach: Springer Science \& Business Media, 2008.

LACY, M. C. D.; REGUZZONI, M.; SANSÒ, F. Real-time cycle slip detection in triple-frequency GNSS. GPS solutions, Springer, v. 16, n. 3, p. 353-362, 2012.

MENDONCA, C. H. M. Detecção e correção de perdas de ciclos para dados GPS de tripla frequência. Dissertação. Faculdade de Ciências e Tecnologia, Universidade Estadual Paulista, Programa de PósGraduação em Ciências Cartográficas, Presidente Prudente, 2019. 106 f.

MONICO, J. F. G. et. al. Acurácia e precisão: revendo os conceitos de forma acurada. Boletim de Ciências Geodésicas, v. 15, n. 3, p. 469-483, 2009.

MONICO, J. F. G. Posicionamento pelo GNSS: descrição, fundamentos e aplicações. São Paulo: Editora UNESP, 2008.

MONICO, J. F. G.; SOUZA, E. M.; MACHADO, C. W. Avaliação de estratégias de detecção e correção de perdas de ciclos na portadora GPS 11. Boletim de Ciências Geodésicas, v. 15, n. 2, p. 178-193, 2009. ISSN 1982-2170.

SEEBER, G. Satellite geodesy: foundations, methods, and applications. 2. ed. New York: Walter de Gruyter, 2003.

SILVA, C. M. Solução das ambiguidades de linhas de bases médias e longas: aplicação no posicionamento baseado em redes. Dissertação. Faculdade de Ciências e Tecnologia, Universidade Estadual Paulista, Programa de Pós-Graduação em Matemática Aplicada e Computacional, Presidente Prudente, 2015. 136 f. 
TEUNISSEN, P.; BAKKER, P. de. Multivariate integer cycle-slip resolution: A single-channel analysis. Springer, 2015.

WOLF, P. R.; GHILANI, C. D. Adjustment computations: statistics and least squares in surveying and GIS. 3. ed. New York: John Wiley \& Sons, 1997.

ZHANG, X.; LI, P. Benefits of the third frequency signal on cycle slip correction. GPS Solutions, Springer, p. 1-10, 2015.

\section{Biografia do autor principal}

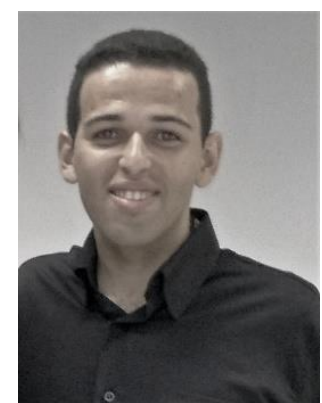

Caio Henrique Chrisóstomo Mendonça, nascido em Presidente Bernardes, São Paulo, no ano de 1994. É Engenheiro Cartógrafo (2017) e Mestre em Ciências Cartográficas (2019) pela Universidade Estadual Paulista (FCT/UNESP), além Técnico Agrimensor (2011) e Técnico em Agropecuária (2011) pela Etec Prof. Dr. Antônio Eufrásio de Toledo. Tem experiência em Geociências, com ênfase em Geodésia, atuando principalmente em projetos de pesquisa com monitoramento de estruturas e posicionamento GNSS (Global Navigation Satellite System). Atualmente atua no mercado de trabalho como Analista de Geoprocessamento.

Esta obra está licenciada com uma Licença Creative Commons Atribuição 4.0 Internacional - CC BY. Esta licença permite que outros distribuam, remixem, adaptem e criem a partir do seu trabalho, mesmo para fins comerciais, desde que lhe atribuam o devido crédito pela criação original. 